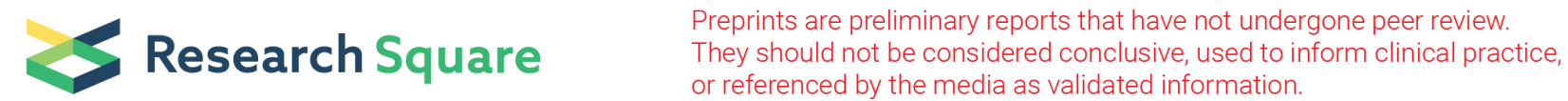

\section{Application of Modified Laparoscopic Radical Hysterectomy in The Treatment of Early Cervical Cancer}

\section{Xuqing Li}

the first affiliated hospital of anhui medical university

\section{Xueting Pei}

the first affiliated hospital of anhui medical university

Hongyan Li

the first affiliated hospital of anhui medical university

Yan Wang

the first affiliated hospital of anhui medical university

Youwei Zhou

the first affiliated hospital of anhui medical university

\section{Zhaolian Wei}

the first affiliated hospital of anhui medical university

\section{Zongzhi Yin ( $\nabla$ dr_yinzongzhi@qq.com )}

The first affiliated hospital of Anhui medical university https://orcid.org/0000-0001-5968-6332

\section{Research}

Keywords: Modified Laparoscopic Radical Hysterectomy, Open Radical Hysterectomy, Early Stage Cervical Cancer, Survival rate

Posted Date: February 23rd, 2021

DOI: https://doi.org/10.21203/rs.3.rs-219139/v1

License: (a) (i) This work is licensed under a Creative Commons Attribution 4.0 International License. Read Full License 


\section{Abstract}

Background: To investigate the safety and efficacy of modified laparoscopic radical hysterectomy (MLRH) in the treatment of early stage cervical cancer by comparing relevant indices of different surgical procedures in patients with early stage cervical cancer.

Methods: Patients with 2014 International Federation of Gynecology and Obstetrics (FIGO) clinical stages IB1 and IIA1 cervical cancer who underwent radical hysterectomy in the Gynecological Department of our hospital from October 2015 to June 2018 were enrolled. Patients were divided into two groups based on the surgical procedure: the open radical hysterectomy $(\mathrm{ORH})$ group $(n=336)$ and MLRH group $(n=302)$. We retrospectively analyzed and compared the clinical characteristics, surgical indices, and survival prognosis between the groups.

Results: Compared to the ORH group, the MLRH group exhibited a longer operative time, normal bladder function recovery time, less intraoperative blood loss volume, and more harvested pelvic lymph nodes $(P<0.05)$. No significant differences were observed in postoperative complications, the 2.5 -year overall survival (OS) rate, 2.5-year disease-free survival (DFS) rate, and recurrence rate between the groups $(P>0.05)$, but the recurrence pattern was significantly different between the groups $(P<0.05)$. Stratified analysis revealed that OS time was shorter in the ORH group than in the MLRH group in patients with stage IB1 and middle invasion $(P<0.05)$. Pathological type was an independent factor for DFS and OS in early stage cervical cancer.

Conclusion: MLRH incorporates a series of measures to prevent tumor spillage. It is a feasible and effective surgical procedure for the treatment of early stage cervical cancer.

Trial registration: Present research is a retrospective study. The study had retrospectively registered on Chinese Clinical Trial Registry (http://www.chictr.org.cn/) and the registered number is ChiCTR1900026306.

\section{Background}

Cervical cancer ranks fourth in the global incidence of malignant tumors in women, and $85 \%$ of cases occur in developing countries. Cervical cancer is one of the leading contributors to cancer-related deaths in women [1]. The incidence of cervical cancer in China is increasing, with 98,900 new cases and 30,500 deaths reported in 2015 [2]. Previous guidelines [3,4] have indicated that both open and minimally invasive approaches are useful for the surgical treatment of 2014 International Federation of Gynecology and Obstetrics (FIGO) early stage cervical cancer, defined as tumors $4 \mathrm{~cm}$ or less that are confined to the cervix [5]. Retrospective studies and meta-analyses have reported that blood loss volume, hospital stay, and postoperative complication rates of the minimally invasive approach are lower than those of open surgery, whereas the 5-year disease-free survival (DFS) and overall survival (OS) rates were similar between the approaches [6, 7]. A meta-analysis by Cao et al. [8] of 2,922 patients with early stage cervical 
cancer (1,230 underwent laparoscopic surgery and 1,692 underwent open surgery) revealed no significant differences in DFS, OS, and recurrence rates between the two groups.

In contrast, a multicenter randomized controlled trial named the trial by Ramirez et al. [9] in 2018 reported that the 4.5-year DFS and OS rates of minimally invasive surgery were lower and the recurrence rate was higher than that of laparotomy in patients with early stage cervical cancer. A retrospective analysis by Melamed et al. [10] reported that the mortality rate was higher in the minimally invasive group than in the open group. According to the SEER database, the 4-year survival rate of minimally invasive surgery decreased at an annual rate of $0.8 \%$ after 2006 . The application of minimally invasive surgery in the treatment of early stage cervical cancer has generated substantial controversy in the gynecology field worldwide.

Notably, there are several limitations of the LACC trial. The trial included 631 patients from 33 centers by recruiting two subjects per center per year, while recurrent cases were mainly concentrated in 14 centers without eliminating bias relevant to research center factors. A large proportion of pathological data were insufficiently detailed in the study, and the effect of surgeons' learning curve on the results was not evaluated. Further, the study did not elaborate on possible factors contributing to the results. Therefore, the advantages and drawbacks of laparoscopic radical hysterectomy (LRH) should be reconsidered. The value of continued use of minimally invasive surgery and/or improvements in operational procedures should be assessed.

At present, the international classical operation of $\mathrm{LRH}$ requires a uterine manipulator to control the uterus. Common surgical procedures include resecting the anterior lobe of the bladder cervical ligament to separate the ureter, resecting the paracervical main sacral ligament, and finally cutting off the vagina in the pelvic cavity under laparoscopic vision. Most scholars have suggested that these procedures lead to tumor spillage and dissemination, which may be a critical reason underpinning the inferior survival outcomes in the minimally invasive approach. When laparoscopic surgery was first applied to colon cancer, potential peritoneal dissemination or metastasis raised substantial attention [11]. Peritoneal dissemination may be due to tumor perforation, operational tumor extrusion, or carbon dioxide $\left(\mathrm{CO}_{2}\right)$ pneumoperitoneum [12], which can be prevented by improved measures [13].

Compared to classic LRH, modified laparoscopic radical hysterectomy (MLRH) incorporates a series of procedures to prevent tumor spillage, such as avoiding the use of a uterine manipulator, initial resection of the paracervical main sacral ligament, cutting off the vagina following stapler closure, and the use of double-distilled water to flush the wound throughout the operation [14]. This approach of surgical resection from the periphery to the center prevents tumor exposure throughout the entire operation, thus reducing the risk of tumor cell metastasis through lymphovascular invasion.

In order to further evaluate the effects of $M L R H$, we retrospectively analyzed the surgical outcomes and survival prognosis of patients subjected to different operative approaches. This study aimed to 
investigate the effectiveness and safety of this novel surgical procedure in the treatment of early stage cervical cancer.

\section{Methods}

\section{Study population}

A total of 638 patients with clinical early stage cervical cancer undergoing radical hysterectomy between October 2015 and June 2018 were included. Patients were divided into the ORH group $(n=336)$ or MLRH group $(n=302)$. The patients selected the surgical approach. The study was approved by the local ethics committee and conducted in accordance with the Declaration of Helsinki as revised in 2013. The specific inclusion and grouping process is presented in the Additional file 1.

Inclusion criteria were as follows: 1) age < 65 years; 2) clinical stages: IB1 and IIA1 (2014 FIGO staging system); and 3) pathological type: squamous cell carcinoma, adenocarcinoma, and adenosquamous carcinoma. Exclusion criteria were as follows: 1 ) age > 65 years; 2) clinical stages: IA1, IA2, IB2, IIA2, IIB, III, and IV (2014 FIGO staging system); 3) histologic types: neuroendocrine carcinoma, clear cell carcinoma, serous carcinoma, small cell carcinoma, or minimal deviation adenocarcinoma; 4) neoadjuvant chemotherapy or radiotherapy before surgery; and 5) severe systemic underlying diseases, immune diseases, mental illness, or other malignancies.

\section{Operative procedure}

Based on Querleu and Morrow's classification, all patients underwent primary type $\mathrm{C}$ hysterectomy and pelvic lymph node dissection (with or without para-aortic lymph node sampling). A subset of patients underwent double appendectomy. All surgeries were conducted in the same manner by a professional certified gynecologist.

\section{ORH group}

After induction of general anesthesia, patients were placed in the supine position. A median longitudinal incision was made from the symphysis pubis to $4 \mathrm{~cm}$ above the navel in the abdomen. The open pelvic cavity was explored, pelvic lymph node was dissected, and extensive hysterectomy was performed.

\section{MLRH group}

Patients adopted the bladder lithotomy position, and five puncture holes were selected after establishing artificial $\mathrm{CO}_{2}$ pneumoperitoneum. A series of measures were incorporated to prevent tumor spillage based on general laparoscopic procedures: 1) avoiding the use a uterine manipulator by using an acutenaculum to push and pull the suture for manipulating the uterus through the fifth puncture; 2) immediately bagging the whole pelvic lymph node after resection; 3 ) resecting the paracervical main sacral ligament and anterior lobe of the bladder cervical ligament first after lymph node dissection; 4) cutting off the vagina after stapler closure and removing the uterus after vaginal pouch suture; 5) 
flushing the open pelvic cavity repeatedly with double-distilled water throughout the operation; and 6) reducing the postoperative pneumoperitoneum pressure by maintaining the pneumoperitoneum pressure at $11 \mathrm{mmHg}$. Specific procedures are depicted in the Additional file 2.

\section{Follow-up}

All patients were followed up every 3 months in the first 2 years, every 6 months in the next $3-5$ years, and then once a year. All patients (except those who were deceased) were followed up until December 31, 2020.

\section{Research variables}

General clinical characteristics included age, body mass index (BMI), clinical stage, histological grading, pathological type, tumor size, intermediate-risk and high-risk factors, and adjuvant therapy. Clinical stage referred to the FIGO staging method. To determine tumor size, preoperative evaluation of the maximum diameter of the tumor measured according to gynecological examination was performed. Intermediaterisk factors included tumor diameter, invasion depth, and vascular tumor thrombus positivity. High-risk factors included positive results for para-uterine and vaginal margin and lymph node metastasis. The pathological results of the patients included in this study suggested that both para-uterine and vaginal margins were negative; thus, they were excluded from the comparison. Adjuvant treatments included radiotherapy, chemotherapy, or concurrent chemoradiation therapy (CCRT) [15], which referred to the Sedlis standard.

\section{Operative outcomes}

Operative outcomes included the operative time, blood loss volume, number of lymph nodes harvested, normal bladder function recovery time, and postoperative complications. Normal bladder function recovery time was determined based on residual urine $<50 \mathrm{ml}$ by ultrasonography. Postoperative complications included urinary system injury, intestinal obstruction, peritonitis, thrombus, perineum and lower extremity edema, symptomatic lymphatic cyst, and delayed incision healing.

Survival prognosis included the 2.5-year DFS rate, 2.5-year OS rate, recurrence rate, recurrence pattern, DFS time, and OS time. Further analysis was completed by stratification of different factors. DFS time referred to the time between surgery and initial recurrence or death due to cancer. OS time referred to the time between surgery and death due to any cause. Recurrence pattern referred to pelvic local single metastasis or distant multiple metastasis. A Cox regression model was used to analyze the prognostic factors of early stage cervical cancer.

\section{Statistical analysis}

For comparisons of continuous variables, the Kruskal-Wallis test was used. For comparisons of categorical variables, the Pearson chi-square test was used. Survival outcomes were compared using Kaplan-Meier analysis with the log-rank test. In both univariate and multivariate analyses, we calculated 
hazard ratios and 95\% confidence intervals using Cox proportional hazards regression models. Statistical analyses were performed using SPSS statistical software (version 25.0). P<0.05 was considered statistically significant.

\section{Results}

\section{Clinical characteristics}

Mean age of patients was higher in the ORH group than in the MLRH group (49 years versus [vs.] 48 years, respectively; $P=0.015$ ). Mean $B M I$ was similar between the groups (ORH and MLRH: $23.15 \mathrm{~kg} / \mathrm{m}^{2}$ and $22.94 \mathrm{~kg} / \mathrm{m}^{2}$, respectively: $\left.P=0.525\right)$. Significant between-group differences were observed in clinical stage $(P<0.001)$ and invasion depth $(P=0.001)$. No significant differences were observed in histological grade, pathological type, vascular tumor emboli positive rate, lymph node metastasis rate, and adjuvant therapy $(P<0.05)$. Specific results are presented in Table 1.

\section{Operative outcomes}

Compared with the ORH group, the MLRH group exhibited a longer operative time (mean: 180 vs. 200 min; $P<0.001$ ), normal bladder function recovery time (mean: $14.6 \mathrm{~d}$ vs. $15.3 \mathrm{~d}$, respectively; $P=0.034$ ), less blood loss volume (mean: $150 \mathrm{~mL}$ vs. $100 \mathrm{~mL} ; \mathrm{P}<0.001$ ), and more harvested lymph nodes (mean: 18 vs. 20; $P=0.005)$. No significant between-group difference was noted in the incidence of postoperative complications (ORH and MLRH: $15.0 \%$ and $10.3 \%$, respectively; $\mathrm{P}=0.085$ ) (Table 2).

\section{Survival prognosis}

No significant between-group differences were observed in the 2.5-year OS, DFS, and recurrence rates $(P>0.05)$. Recurrence pattern was significantly different between the groups $(P=0.019)$. The ORH group mainly exhibited distant multiple metastases $(P=87.5 \%, 2 / 16)$, whereas the MLRH group mainly exhibited local single metastasis $(63.6 \%, 7 / 11)$. Specific data are presented in Table 3.

\section{Subgroup comparisons of DFS and OS}

Subjects were divided into subgroups based on factors such as the surgical approach, clinical stage, histological grade, pathological type, tumor size, invasion depth, vascular tumor thrombus, and lymph node metastasis. As shown in Table 4, DFS time was shorter in the adenocarcinoma and adenosquamous carcinoma groups than in the squamous carcinoma group (median: 58.00 months vs. 47.300 months vs. 60.561 months; $P<0.05$, Additional file 3). DFS time was shorter in the lymph node metastasis-positive group than in the negative group (median: 57.645 months vs. 60.499 months; $P=0.005)$. No significant difference was observed in DFS time between the other subgroups $(P>0.05)$.

OS time was shorter in the ORH group than in the MLRH group (median: 60.506 months vs. 61.684 months, $\mathrm{P}=0.014$ ). OS time was shorter in the adenocarcinoma and adenosquamous carcinoma groups than in the squamous carcinoma group (median: 59.514 months vs. 47.329 months vs. 61.534 months; 
$\mathrm{P}<0.05$, Additional file 3). OS time of patients with middle invasion (median: 59.744 months vs. 61.720 months; $\mathrm{P}<0.05$; Additional file 4) and lymph node metastasis positivity was shorter than that of those without (59.483 months vs. 61.383 months, respectively; $P=0.010$ ). No significant difference was noted in OS time between the other subgroups $(P>0.05)$.

\section{Stratified comparison of survival prognosis by different surgical approach}

The prognosis of the ORH and MLRH groups was stratified and compared based on factors such as the clinical stage, tumor size, invasion depth, and adjuvant treatment. As shown in Table 5, OS time was shorter in the ORH group than in the MLRH group for patients with stage IB1 (median: 60.667 months vs. 61.818 months; $\mathrm{P}=0.011$ ). OS time differed significantly between the $\mathrm{ORH}$ and $\mathrm{MLRH}$ groups for patients with middle invasion ( $P=0.043)$. OS rate was lower in the ORH group than in the MLRH group, and it decreased over time. No deaths occurred in the MLRH group. Specific results are shown in Additional file

\section{$5,6,7,8$.}

\section{Analysis of prognostic factors in patients with cervical cancer}

The results of univariate and multivariate analyses using Cox proportional hazards regression models are presented in Table 6. Multivariate analyses identified pathological type and lymph node metastasis as important factors of DFS in patients with early stage cervical cancer. In particular, factors such as adenocarcinoma, adenosquamous carcinoma, and lymph node metastasis significantly reduced DFS ( $P=0.008, P=0.019$, and $P=0.023$, respectively). Invasion depth was a possible factor but was not statistically significant $(P=0.087)$. A critical factor of $O S$ in patients with early stage cervical cancer was pathological type. Specifically, adenocarcinoma and adenosquamous carcinoma significantly reduced OS ( $P=0.003$ and $P<0.001$, respectively). Surgical approach and invasion depth were possible predictive factors $(P=0.056)$.

\section{Discussion}

Our findings suggested that general clinical characteristics of both groups were largely similar. Differences in age, clinical stage, and invasion depth were noted. We subsequently performed stratified analysis of the clinical stage and invasion depth by different approaches. Operative time was longer, blood volume was lower, and number of lymph nodes harvested was higher in the MLRH group than in the ORH group, which is consistent with findings of previous reports [16-17]. These results highlight the advantages of laparoscopic surgery. The microscopic multi-angle vision of the laparoscope enables clearer exposure of tissue anatomy, which is conducive to a more meticulous operation. The recovery time of normal bladder function was longer in the MLRH group than in the ORH group, which could be due to electrothermal conductive nerve injury caused by energy instruments [18]. Further improvements in surgical procedures are required to minimize the effects of heat injury on bladder function. The paracervical tissue at and above the deep vein level should be clipped with vascular clamps and cut off with non-energy surgical scissors to maximally preserve the pelvic autonomic nerve, especially the bladder plexus [19]. No significant between-group difference was noted in the incidence of postoperative 
complications, consistent with the results of the LACC test [9]. In theory, the incidence of complications should be lower for MLRH, and this issue should be pursued by future studies.

The LACC trial demonstrated that the 3-year DFS rates of the minimally invasive and open groups were $91.2 \%$ and $97.1 \%$, respectively $(P<0.05)$, whereas the 3 -year OS rates were $93.8 \%$ and $99.0 \%$, respectively $(P<0.05)$. This indicated that minimally invasive radical hysterectomy was associated with lower DFS and OS in patients with early stage cervical cancer. Our results revealed no significant differences in the 2.5year OS rate, DFS rate, and recurrence rate between the MLRH and ORH groups. This may benefit from additional procedures for preventing tumor cell spillage and micrometastasis in MLRH [20]. Kanao [21] compared the outcomes of laparoscopic surgery with the no-look no-touch technique to those of open surgery in the treatment of early stage cervical cancer. No significant between-group differences were noted in the 2.5 -year DFS ( $94.4 \%$ vs. $90.9 \%, P=0.591)$ and OS (100\% vs. $96.5 \%, P=0.188)$, consistent with our results.

In the LACC trial, the recurrence pattern of the laparoscopic group manifested predominantly as multiple pelvic recurrence, distinct to that of the open group. This may be related to tumor cell spillover and dissemination during the operation. However, our study demonstrated that there was more pelvic local recurrence in the MLRH group than in the $\mathrm{ORH}$ group and more distant multiple recurrences in the $\mathrm{ORH}$ group than in the MLRH group. Possible reasons for this include the improved procedures of laparoscopic surgery having a definite effect on the prevention of tumor cell spillage and repeated extrusion of cervical lesions during open surgery leading to tumor micrometastasis [22]. In particular, when the cervical tumor is difficult to expose, the cervix will inevitably be extruded during open operation. In the MLRH, we exposed the operating space fully by suspension of the uterus, which ensured that the tumor was completely free of touch and exposure.

DFS was shorter in the adenocarcinoma and adenosquamous carcinoma groups than in the squamous carcinoma group, whereas DFS was shorter in the lymph node metastasis-positive group than in the negative group, corresponding to the prognostic risk factors of early stage cervical cancer [23, 24]. OS was short in the adenocarcinoma, adenocarcinoma, and lymph node metastasis-positive groups as well as in the ORH and deep invasion depth groups. The different surgical approaches and invasion depth influenced OS but not DFS, which may be compensated for by better responses to adjuvant therapy with difference recurrence patterns.

The clinical stage of patients with cervical cancer in our study was determined based on the 2014 FIGO staging criteria [25]. The difference between IB1 and IB2 stages is whether the tumor diameter exceeded 4 $\mathrm{cm}$. IB stages are divided into stage IB1 (tumor diameter $\leq 2 \mathrm{~cm}$ ), stage IB2 (tumor diameter 2-4 cm), and stage IB3 (tumor diameter $>4 \mathrm{~cm}$ ) based on the latest 2018 FIGO stage [26]. This indicates that tumor size is closely related to the survival prognosis of cervical cancer [27]. At the 2018 Annual Meeting of the American Clinical Oncologic Society, scholars proposed that OS was not lower for laparoscopic surgery than for open surgery in patients with cervical cancer of diameter $<2 \mathrm{~cm}[28]$. Kim et al. $[29,30]$ reported similar conclusions in their recent studies. To eliminate the effects of adjuvant therapy, Paik et 
al. [31] selected IB1 cervical cancer patients without adjuvant treatment for retrospective analysis, and reported that DFS was significantly lower in laparoscopic radical hysterectomy than in open surgery.

Accordingly, our data were further stratified based on clinical stage, tumor diameter, and adjuvant treatment. For patients with stage IB1 and middle invasion, OS time was longer in the MLRH group than in the ORH group, highlighting the advantages of MLRH for survival outcomes in these subgroups [32, 33]. No significant differences were observed in DFS and OS between the two subgroups with a tumor diameter 2-4 cm and who received adjuvant therapy, although the P-value of OS approximated 0.05 $(P=0.056 ; P=0.068)$. The lesions of patients with a larger tumor diameter and adjuvant therapy were more severe, which may have increased the risk of tumor contact and squeezing in open surgery. Larger samples are required for confirmation in the future.

\section{Conclusion}

MLRH incorporates a series of measures to prevent tumor spillage. It is a feasible and effective surgical procedure for the treatment of early stage cervical cancer.

\section{Strengths}

The strengths of this study are as follows: 1) the number of subjects included in the study was considerable because of the large population base in China and relatively large number of patients with cervical cancer; 2) patients in the MLRH group received novel surgical methods, adding a series of innovative procedures to prevent tumor extravasation, and the surgeries were performed by the same gynecological team, thereby avoiding the influence of surgeons' learning curve on the results [34-35]; and 3) to reduce confounding bias as much as possible, the results were comprehensively compared and analyzed based on various factors.

\section{Limitations}

This study has several limitations. First, it was a retrospective study, and the included subjects were not randomized, potentially leading to sample selection bias. These factors are difficult to evaluate in retrospective analysis. Second, patients who received surgical treatment in our center were used as the sample, and the influence of institutional heterogeneity, especially differences in the surgical operation on the results, could not be analyzed [36]. Third, the follow-up time was relatively short. MRLH has mainly been performed since 2015. The median follow-up time was 42 months (30-62 months), which could be extended to more than 5 years later. In the future, the use of energy instruments can be further reduced to avoid electric heat damage of the pelvic autonomic nerve. Larger prospective randomized controlled studies are required to provide stronger evidence for the feasibility and safety of MLRH.

\section{Declarations}

\section{Ethics approval and consent to participate}


The research protocol and procedures were reviewed and approved by the First Affiliated Hospital of Anhui Medical University Ethics Committee for the Protection of Human Subjects in Research (No. PJ2019-12-05).

\section{Consent for publication}

N/A

\section{Availability of data and materials}

The datasets used and/or analysed during the current study are available from the corresponding author on reasonable request.

\section{Competing interests}

The authors declare that they have no competing interests.

\section{Funding}

This work was partly funded by the National Natural Science Foundation of China (No. 82071679) and the University Natural Science Foundation of Anhui Educational Committee (KJ2018A0188).

\section{Authors' contributions}

Dr. Yin Zongzhi contributed to Conceptualization, Formal analysis, Funding acquisition, Writing - review \& editing. Dr. Wei Zhaolian contributed to Data curation, Investigation, Methodology, Project administration, and Supervision. They have equal contribution as corresponding author. Dr. Li Xuqing contributed to Conceptualization, Methodology, Validation, Formal analysis and investigation, Writing -original draft preparation. Dr. Pei Xueting contributed to Formal analysis and investigation, Writing - original draft preparation. Dr. Li Hongyan, Dr. Wang Yan and Dr. Zhou Youwei contributed to Methodology, Formal analysis and investigation, and Resources.

\section{Acknowledgements}

N/A

\section{References}

1. Jemal A, Bray F, Center MM, Ferlay J, Ward E, Forman D. Global cancer statistics. CA Cancer J Clin. 2011;61:69-90.

2. Chen W, Zheng R, Baade PD, Zhang S, Zeng H, Bray F, et al. Cancer statistics in China, 2015. CA Cancer J Clin. 2016;66:115-32.

3. NCCN. Clinical practice guidelines in oncology (NCCN Guidelines $\left.{ }^{\circledR}\right)$. Cervical Cancer. Version 2019; 2018. 
4. Marth C, Landoni F, Mahner S, McCormack M, Gonzalez-Martin A, Colombo N, et al. Cervical cancer: ESMO clinical practice guidelines for diagnosis, treatment and follow-up. Ann Oncol. 2017;28(Suppl 4):72-83.

5. Costales A, Michener C, Escobar-Rodriguez PF. Radical trachelectomy for early stage cervical cancer. Curr Treat Options Oncol. 2018;19:75.

6. Lee EJ, Kang H, Kim DH. A comparative study of laparoscopic radical hysterectomy with radical Open hysterectomy for early-stage cervical cancer: a long-term follow-up study. Eur J Obstet Gynecol Reprod Biol. 2011;156:83-6.

7. Nam J-H, Park J-Y, Kim D-Y, Kim J-H, Kim Y-M, Kim Y-T. Laparoscopic versus open radical hysterectomy in early-stage cervical cancer: long-term survival outcomes in a matched cohort study. Ann Oncol. 2012;23:903-11.

8. Cao T, Feng Y, Huang Q, Wan T, Liu J. Prognostic and safety roles in laparoscopic versus Open radical hysterectomy in cervical cancer: a meta-analysis. J Laparoendosc Adv Surg Tech A. 2015;25:990-8.

9. Ramirez PT, Frumovitz M, Pareja R, Lopez A, Vieira M, Ribeiro R, et al. Minimally invasive versus open radical hysterectomy for cervical cancer. N Engl J Med. 2018;379:1895-904.

10. Melamed A, Margul DJ, Chen L, Keating NL, Del Carmen MG, Yang J, et al. Survival after minimally invasive radical hysterectomy for early-stage cervical cancer. N Engl J Med. 2018;379:1905-114.

11. Bertagnolli MM, DeCosse JJ. Laparoscopic colon resection for cancer-an unfavorable view. Adv Surg. 1996;29:155-64.

12. Kong TW, Chang SJ, Piao X, Paek J, Lee Y, Lee EJ, et al. Patterns of recurrence and survival after Open versus laparoscopic/robotic radical hysterectomy in patients with early cervical cancer. J Obstet Gynaecol Res. 2016;42:77-86.

13. Balli JE, Franklin ME, Almeida JA, Glass JL, Diaz JA, Reymond M. How to prevent port-site metastases in laparoscopic colorectal surgery. Surg Endosc. 2000;14:1034-6.

14. Shiozaki A, Ichikawa D, Kosuga T, Marunaka Y, Otsuji E. Regulation of osmolality for cancer treatment. J Physiol Sci. 2017;67:353-60.

15. Li M, Hu M, Wang Y, Yang X. Adjuvant chemoradiotherapy versus radiotherapy in cervical cancer patients with intermediate-risk factors: A systematic review and meta-analysis. Eur J Obstet Gynecol Reprod Biol. 2019;238:1-6.

16. Wang W, Chu HJ, Shang CL, Gong X, Liu TY, Zhao YH, et al. Long-term oncological outcomes after laparoscopic versus open radical hysterectomy in stage IA2 to IIA2 cervical cancer: a matched cohort study. Int J Gynecol Cancer. 2016;26:1264-73.

17. Wang YZ, Deng L, Xu HC, Zhang Y, Liang ZQ. Laparoscopy versus laparotomy for the management of early stage cervical cancer. BMC Cancer. 2015;15:928.

18. Bogani G, Rossetti D, Ditto A, Martinelli F, Chiappa V, Leone C, et al. Minimally in vasive surgery improves short-term outcomes of nerve-sparing radical hysterectomy in patients with cervical cancer: a propensity-matched analysis with open surgery. J Gynecol Oncol. 2019;30:e27. 
19. Kietpeerakool C, Aue-Aungkul A, Galaal K, Ngamjarus C, Lumbiganon P. Nerve-sparing radical hysterectomy compared to standard radical hysterectomy for women with early stage cervical cancer (stage la2 to lla). Cochrane Database Syst Rev. 2019;2:CD012828.

20. Wei XQ, Ma Y, Chen Y, Liu X, Zhao M, Zhou LW. Laparoscopic surgery for early cervical squamous cell carcinoma and its effect on the micrometastasis of cancer cells.Medicine. (Baltimore). 2018;97:e11921.

21. Kanao H, Matsuo K, Aoki Y, Tanigawa T, Nomura H, Okamoto S, et al. Feasibility and outcome of total laparoscopic radical hysterectomy with no-look no-touch technique for FIGO IB1 cervical cancer. J Gynecol Oncol. 2019;30:e71.

22. O'Leary D, O'Leary E, Foley N, Cotter TG, Wang JH, Redmond HP. Effects of surgery on the cancer stem cell niche. Eur J Surg Oncol. 2016;42:319-25.

23. Takekuma M, Kasamatsu Y, Kado N, Kuji S, Tanaka A, Takahashi N, et al. The issues regarding postoperative adjuvant therapy and prognostic risk factors for patients with stage $\mathrm{I}-\mathrm{Il}$ cervical cancer: A review. J Obstet Gynaecol Res. 2017;43:617-26.

24. Kim H, Cho WK, Kim YJ, Kim YS, Park W. Significance of the number of high-risk factors in patients with cervical cancer treated with radical hysterectomy and concurrent chemoradiotherapy. Gynecol Oncol. 2020;157:423-8.

25. FIGO Committee on Gynecologic Oncology. FIGO staging for carcinoma of the vulva, cervix, and corpus uteri. Int J Gynaecol Obstetr. 2014;125:97-8.

26. Matsuo K, Machida H, Mandelbaum RS, Konishi I, Mikami M. Validation of the 2018 FIGO cervical cancer staging system. Gynecol Oncol. 2019;152:87-93.

27. Canaz E, Ozyurek ES, Erdem B, Talmac MA, Ozaydin IY, Akbayir O, et al. Preoperatively assessable clinical and pathological risk factors for parametrial involvement in surgically treated FIGO stage IBIIA cervical cancer. Int J Gynecol Cancer. 2017;27:1722-8.

28. Margul DJ, Yang J, Seagle BL, Kocherginsky M, Shahabi S. Outcomes and costs of open, robotic and laparoscopic radical hysterectomy for stage IB1 cervical cancer. J Clin Oncol. 2018;36:5502.

29. Kim SI, Cho JH, Seol A, Im Kim Y, Lee M, Kim HS, et al. Comparison of survival outcomes between minimally invasive surgery and conventional open surgery for radical hysterectomy as primary treatment in patients with stage IB1-IIA2 cervical cancer. Gynecol Oncol. 2019;153:3-12.

30. Kim SI, Lee M, Lee S, Suh DH, Kim HS, Kim K, et al. Impact of laparoscopic radical hysterectomy on survival outcome in patients with FIGO stage IB cervical cancer: A matching study of two institutional hospitals in Korea. Gynecol Oncol. 2019;155:75-82.

31. Paik ES, Lim MC, Kim MH, Nie D, Li Z. Comparison of laparoscopic and open radical hysterectomy in early stage cervical cancer patients without adjuvant treatment: Ancillary analysis of a Korean Gynecologic Oncology Group Study (KGOG 1028). Gynecol Oncol. 2019;154:547-53.

32. Matsuo K, Mabuchi S, Okazawa M, Matsumoto Y, Tsutsui T, Fujita M, et al. Utility of risk-weighted surgical-pathological factors in early-stage cervical cancer. Br J Cancer. 2013;108:1348-57. 
33. Margul DJ, Yang J, Seagle BL, Kocherginsky M, Shahabi S. Outcomes and costs of open, robotic, and laparoscopic radical hysterectomy for stage IB1 cervical cancer. J Clin Oncol. 2018;36:5502.

34. Kim S, Min KJ, Lee S, Hong JH, Song JY, Lee JK, et al. Learning curve could affect oncologic outcome of minimally invasive radical hysterectomy for cervical cancer. Asian J Surg. 2021;44:17480.

35. Liu Y, Li L, Wu M, Ma S, Tan X, Zhong S, et al. The impact of the surgical routes and learning curve of radical hysterectomy on the survival outcomes in stage IB cervical cancer: A retrospective cohort study. Int J Surg. 2019;68:72-7.

36. Suh DH, Cho HY, Kim K, No JH, Kim YB. Matched-case comparisons in a single institution to determine critical points for inexperienced surgeons' successful performances of laparoscopic radical hysterectomy versus open radical hysterectomy in stage IA2-IIA cervical cancer. PLoS One. 2015;10:e0131170.

\section{Tables}

Table 1. Clinical characteristics of the patients 


\begin{tabular}{|c|c|c|c|}
\hline Characteristic & $\mathrm{ORH}$ & MLRH & $P$-value \\
\hline Total & 336 & 302 & \\
\hline Patients lost to follow-up (n) & 15 & 11 & \\
\hline Patients followed up with (n) & 321 & 291 & \\
\hline Age (y) & $49(43-54)$ & $48(41-52)$ & 0.015 \\
\hline $\mathrm{BMI}\left(\mathrm{kg} / \mathrm{m}^{2}\right)$ & $23.15(21.23-24.93)$ & $22.94(20.76-25.39)$ & 0.525 \\
\hline Histological grading (\%) & & & 0.146 \\
\hline G1 & $23(7.2)$ & $45(15.5)$ & \\
\hline G2 & $173(53.9)$ & $136(46.7)$ & \\
\hline G3 & $125(38.9)$ & $110(37.8)$ & \\
\hline Pathological type (\%) & & & 0.700 \\
\hline Squamous cell carcinoma & $262(81.6)$ & $233(80.1)$ & \\
\hline Adenocarcinoma & $55(17.1)$ & $52(17.9)$ & \\
\hline Adenosquamous carcinoma & $4(1.2)$ & $6(2.1)$ & \\
\hline FIGO stage (\%) & & & $<0.001$ \\
\hline IB1 & $265(82.6)$ & 269 (92.4) & \\
\hline IIA1 & $56(17.4)$ & $22(7.6)$ & \\
\hline Invasion depth (\%) & & & 0.001 \\
\hline Inner $1 / 3$ & $119(37.1)$ & $142(48.8)$ & \\
\hline Middle 1/3 & $116(36.1)$ & 97 (33.3) & \\
\hline Deep 1/3 & $86(26.8)$ & $52(17.9)$ & \\
\hline Vascular tumor embolus (\%) & & & 0.325 \\
\hline Negative & $243(75.7)$ & $230(79.0)$ & \\
\hline Positive & $78(24.3)$ & $61(21.0)$ & \\
\hline Lymph node metastasis (\%) & & & 0.143 \\
\hline Negative & $258(80.4)$ & 247 (84.9) & \\
\hline Positive & $63(19.6)$ & $44(15.1)$ & \\
\hline Adjuvant therapy (\%) & & & 0.058 \\
\hline None & $163(50.8)$ & 179 (61.5) & \\
\hline
\end{tabular}




\begin{tabular}{|lll|}
\hline Radiotherapy & $35(10.9)$ & $28(9.6)$ \\
\hline Chemotherapy & $22(6.9)$ & $14(4.8)$ \\
\hline CCRT & $101(31.5)$ & $70(24.1)$ \\
\hline
\end{tabular}

FIGO, 2014 International Federation of Gynecology and Obstetrics; CCRT, concurrent chemoradiation therapy; $\mathrm{ORH}$, open radical hysterectomy; MLRH, modified laparoscopic radical hysterectomy

Table 2. Operating outcomes of patients

\begin{tabular}{|llll|}
\hline Characteristic & ORH & MLRH & $P$-value \\
\hline Operative time $(\mathrm{min})$ & $180(150-220)$ & $200(180-240)$ & $<0.001$ \\
\hline Blood loss volume $(\mathrm{mL})$ & $150(100-200)$ & $100(50-100)$ & $<0.001$ \\
\hline Lymph nodes harvested $(\mathrm{n})$ & $18(14-22)$ & $20(15-24)$ & 0.005 \\
\hline Normal bladder function recovery time (d) & $14.6(13-15)$ & $15.3(13-15)$ & 0.034 \\
\hline Postoperative complications (\%) & & & 0.085 \\
\hline None & $273(85.0)$ & $261(89.7)$ & \\
\hline Yes & $48(15.0)$ & $30(10.3)$ & \\
\hline Urinary damage & $5(1.6)$ & $4(1.4)$ & 1.000 \\
\hline Intestinal obstruction & $9(2.8)$ & $7(2.4)$ & 0.758 \\
\hline Thrombus & $3(0.9)$ & $2(0.7)$ & 1.000 \\
\hline Symptomatic lymphatic cyst & $10(3.1)$ & $5(1.7)$ & 0.264 \\
\hline Delayed incision healing & $7(2.2)$ & $3(1.0)$ & 0.423 \\
\hline Perineal and lower limb edema & $8(2.5)$ & $6(2.1)$ & 0.722 \\
\hline Peritonitis & $14(4.4)$ & $11(3.8)$ & 0.717 \\
\hline
\end{tabular}

$\mathrm{ORH}$, open radical hysterectomy; MLRH, modified laparoscopic radical hysterectomy

Table 3. Survival and recurrence outcome of patients 


\begin{tabular}{|llll|}
\hline Characteristic & ORH & MLRH & P-value \\
\hline 2.5-year OS rate (\%) & $313(97.5)$ & $289(99.3)$ & 0.150 \\
\hline 2.5-year DFS rate (\%) & $310(96.6)$ & $281(96.6)$ & 0.995 \\
\hline 2.5-year recurrence rate (\%) & $11(3.4)$ & $10(3.4)$ & 0.995 \\
\hline Recurrence pattern & & & 0.019 \\
\hline Local single (\%) & $2(12.5)$ & $7(63.6)$ & \\
\hline Distant multiple (\%) & $14(87.5)$ & $4(36.4)$ & \\
\hline
\end{tabular}

$\mathrm{ORH}$, open radical hysterectomy; MLRH, modified laparoscopic radical hysterectomy; OS, overall survival; DFS, disease-free survival

Table 4. Subgroup comparison of DFS and OS in cervical cancer 


\begin{tabular}{|c|c|c|c|c|c|}
\hline Characteristic & Number & $\begin{array}{l}\text { DFS time } \\
\text { (months) }\end{array}$ & $\begin{array}{l}P- \\
\text { value }\end{array}$ & $\begin{array}{l}\text { OS time } \\
\text { (months) }\end{array}$ & $P$-value \\
\hline Surgical approach & & & 0.506 & & 0.014 \\
\hline $\mathrm{ORH}$ & 321 & 59.830 & & 60.506 & \\
\hline MLRH & 291 & 60.201 & & 61.684 & \\
\hline FIGO stage & & & 0.136 & & 0.071 \\
\hline IB1 & 534 & 60.217 & & 61.241 & \\
\hline$\| \mathrm{A} 1$ & 78 & 58.563 & & 59.783 & \\
\hline Histological grade & & & 0.238 & & 0.878 \\
\hline G1 & 68 & 61.235 & & 61.279 & \\
\hline G2 & 309 & 60.366 & & 60.951 & \\
\hline G3 & 235 & 58.251 & & 60.155 & \\
\hline Pathological type & & & 0.003 & & $<0.001$ \\
\hline Squamous cell carcinoma & 495 & 60.561 & & 61.534 & \\
\hline Adenocarcinoma & 107 & 58.000 & & 59.514 & \\
\hline $\begin{array}{l}\text { Adenosquamous } \\
\text { carcinoma }\end{array}$ & 10 & 47.300 & & 47.329 & \\
\hline Tumor size & & & 0.180 & & 0.192 \\
\hline$<20 \mathrm{~mm}$ & 185 & 60.649 & & 61.551 & \\
\hline $20-40 \mathrm{~mm}$ & 427 & 59.715 & & 60.838 & \\
\hline Invasion depth & & & 0.100 & & 0.020 \\
\hline Inner $1 / 3$ & 261 & 60.788 & & 61.720 & \\
\hline Middle 1/3 & 213 & 59.932 & & 61.069 & \\
\hline Deep 1/3 & 138 & 58.566 & & 59.744 & \\
\hline Vascular tumor emboli & & & 0.061 & & 0.059 \\
\hline Negative & 473 & 60.380 & & 61.320 & \\
\hline Positive & 139 & 58.684 & & 60.084 & \\
\hline Lymph node metastasis & & & 0.005 & & 0.010 \\
\hline Negative & 505 & 60.499 & & 61.383 & \\
\hline Positive & 107 & 57.645 & & 59.483 & \\
\hline
\end{tabular}


FIGO, 2014 International Federation of Gynecology and Obstetrics; ORH, open radical hysterectomy; $\mathrm{MLRH}$, modified laparoscopic radical hysterectomy; OS, overall survival; DFS, disease-free survival

Table 5. Stratified comparison of survival prognosis by surgical approach 


\begin{tabular}{|c|c|c|c|c|c|}
\hline \multirow[t]{2}{*}{ Stratification factor } & \multirow{2}{*}{$\begin{array}{l}\text { Surgical } \\
\text { approach }\end{array}$} & \multicolumn{2}{|l|}{ DFS } & \multicolumn{2}{|l|}{ OS } \\
\hline & & $\begin{array}{l}\text { Time } \\
\text { (months) }\end{array}$ & $\begin{array}{l}P \text { - } \\
\text { value }\end{array}$ & $\begin{array}{l}\text { Time } \\
\text { (months) }\end{array}$ & $P$-value \\
\hline \multirow[t]{3}{*}{ Clinical stage, IB1 } & & & 0.513 & & 0.011 \\
\hline & $\mathrm{ORH}$ & 60.038 & & 60.667 & \\
\hline & MLRH & 60.412 & & 61.818 & \\
\hline \multirow[t]{3}{*}{ Clinical stage, IIA1 } & & & 0.713 & & 0.941 \\
\hline & $\mathrm{ORH}$ & 58.974 & & 59.735 & \\
\hline & MLRH & 53.091 & & 55.273 & \\
\hline \multirow{3}{*}{$\begin{array}{l}\text { Tumor size } \\
<2 \mathrm{~cm}\end{array}$} & & & 0.750 & & 0.132 \\
\hline & $\mathrm{ORH}$ & 60.851 & & - & \\
\hline & MLRH & 58.531 & & - & \\
\hline \multirow[t]{3}{*}{$2-4 \mathrm{~cm}$} & & & 0.441 & & 0.056 \\
\hline & $\mathrm{ORH}$ & 59.459 & & 60.315 & \\
\hline & $\mathrm{MLRH}$ & 60.065 & & 61.523 & \\
\hline \multirow[t]{3}{*}{ Inner $1 / 3$ invasion } & & & 0.556 & & 0.122 \\
\hline & $\mathrm{ORH}$ & 60.555 & & - & \\
\hline & $\mathrm{MLRH}$ & 61.000 & & - & \\
\hline \multirow[t]{3}{*}{ Middle $1 / 3$ invasion } & & & 0.311 & & 0.043 \\
\hline & $\mathrm{ORH}$ & 59.372 & & - & \\
\hline & MLRH & 58.639 & & - & \\
\hline \multirow[t]{3}{*}{ Deep 1/3 invasion } & & & 0.350 & & 0.674 \\
\hline & $\mathrm{ORH}$ & 59.368 & & 59.520 & \\
\hline & MLRH & 52.801 & & 55.423 & \\
\hline \multirow{3}{*}{$\begin{array}{l}\text { No adjuvant } \\
\text { treatment }\end{array}$} & & & 0.326 & & 0.155 \\
\hline & $\mathrm{ORH}$ & 60.017 & & 61.115 & \\
\hline & MLRH & 58.693 & & 59.737 & \\
\hline \multirow[t]{3}{*}{ Adjuvant treatment } & & & 0.885 & & 0.068 \\
\hline & $\mathrm{ORH}$ & 59.640 & & 59.863 & \\
\hline & MLRH & 59.510 & & 61.616 & \\
\hline
\end{tabular}


$\mathrm{ORH}$, open radical hysterectomy; MLRH, modified laparoscopic radical hysterectomy; OS, overall survival; DFS, disease-free survival

Table 6. Cox regression analysis of factors affecting DFS and OS in early stage cervical cancer 


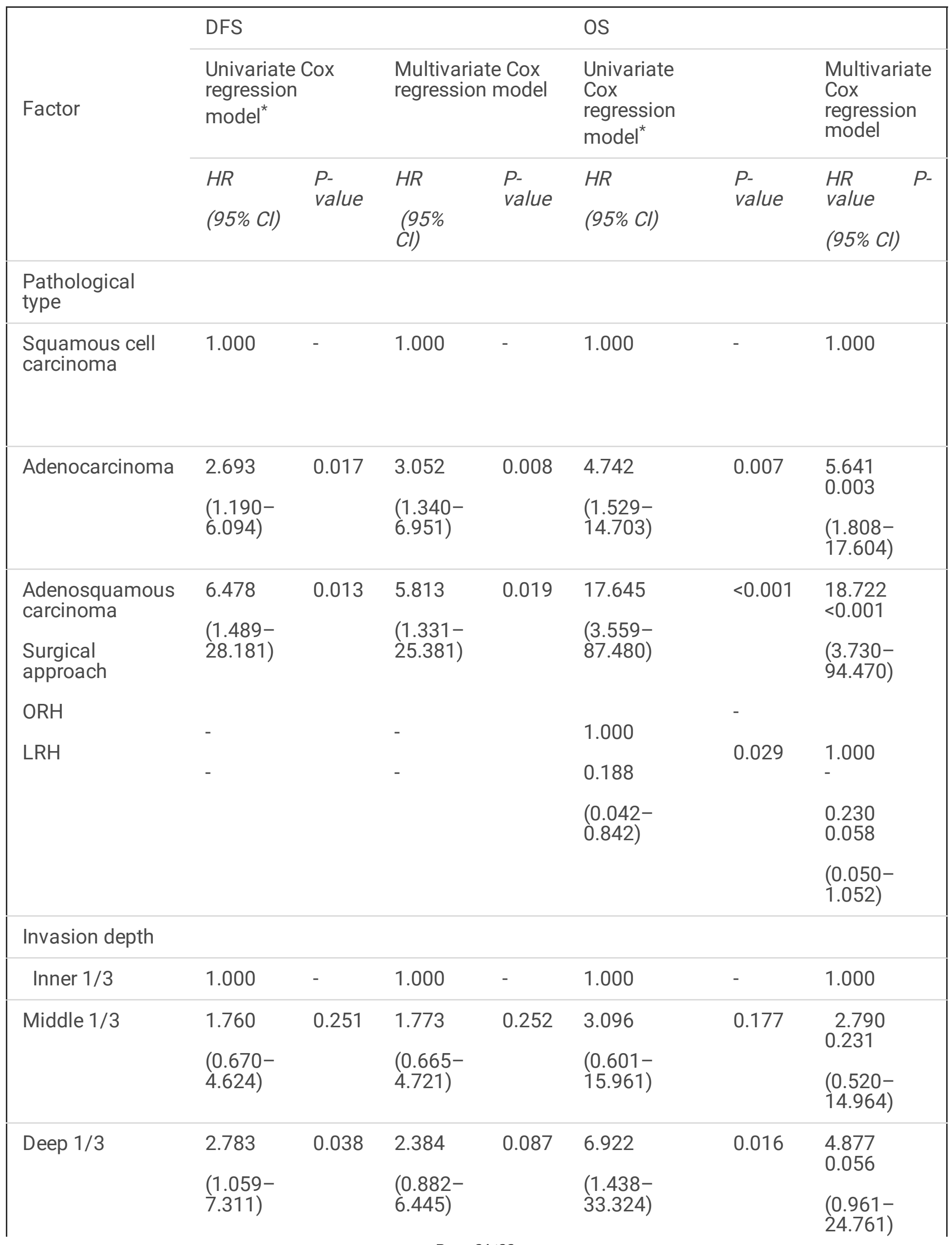


Lymph node metastasis

\begin{tabular}{|c|c|c|c|c|c|c|c|}
\hline Negative & 1.000 & - & 1.000 & - & 1.000 & - & $\begin{array}{l}1.000 \\
-\end{array}$ \\
\hline Positive & $\begin{array}{l}2.885 \\
(1.321- \\
6.301)\end{array}$ & 0.008 & $\begin{array}{l}2.547 \\
(1.139- \\
5.694)\end{array}$ & 0.023 & $\begin{array}{l}3.651 \\
(1.267- \\
10.522)\end{array}$ & 0.017 & $\begin{array}{l}2.435 \\
0.110 \\
(0.817- \\
7.260)\end{array}$ \\
\hline
\end{tabular}

*Univariate Cox regression model analysis only revealed $\mathrm{P}<0.05$.

$\mathrm{ORH}$, open radical hysterectomy; MLRH, modified laparoscopic radical hysterectomy; OS, overall survival; DFS, disease-free survival; $\mathrm{HR}$, hazard ratio; $\mathrm{Cl}$, confidence interval.

\section{Supplementary Files}

This is a list of supplementary files associated with this preprint. Click to download.

- Additionalfile1.jpg

- Additionalfile3.docx

- Additionalfile4.docx

- Additionalfile5.jpg

- Additionalfile6.jpg

- Additionalfile7.jpg

- Additionalfile8.jpg 